TRANSACTIONS OF THE

AMERICAN MATHEMATICAL SOCIETY

Volume 360, Number 10, October 2008, Pages 5603-5627

S 0002-9947(08)04511-X

Article electronically published on May 21, 2008

\title{
SAMPLING IN PALEY-WIENER SPACES ON COMBINATORIAL GRAPHS
}

\author{
ISAAC PESENSON
}

\begin{abstract}
A notion of Paley-Wiener spaces on combinatorial graphs is introduced. It is shown that functions from some of these spaces are uniquely determined by their values on some sets of vertices which are called the uniqueness sets. Such uniqueness sets are described in terms of Poincare-Wirtingertype inequalities. A reconstruction algorithm of Paley-Wiener functions from uniqueness sets which uses the idea of frames in Hilbert spaces is developed. Special consideration is given to the $n$-dimensional lattice, homogeneous trees, and eigenvalue and eigenfunction problems on finite graphs.
\end{abstract}

\section{IntRoduction AND MAIN RESUlts}

The goal of the paper is to develop a sampling theory of Paley-Wiener functions (bandlimited functions) on combinatorial graphs. It is shown that functions which involve only "low" frequencies can be perfectly reconstructed from their values on some subsets of vertices, but "high" frequencies are uniquely determined only by their values at all vertices. Note that on a continuous manifold for any frequency one can construct a sampling set of sufficient density which will allow reconstruction of that frequency.

Let us recall some basic facts from the classical sampling theory. A function $f \in L_{2}(\mathbb{R})$ is called $\omega$-bandlimited if its $L_{2}$-Fourier transform

$$
\hat{f}(t)=\int_{-\infty}^{+\infty} f(x) e^{-2 \pi i x t} d x
$$

has support in $[-\omega, \omega]$. The Paley-Wiener theorem states that $f \in L_{2}(\mathbb{R})$ is $\omega$ bandlimited if and only if $f$ is an entire function of exponential type not exceeding $2 \pi \omega$. $\omega$-bandlimited functions form the Paley-Wiener class $P W_{\omega}(\mathbb{R})$ and are often called Paley-Wiener functions. The classical sampling theorem says that if $f$ is $\omega$-bandlimited, then $f$ is completely determined by its values at points $j / 2 \omega, j \in \mathbb{Z}$, and can be reconstructed in a stable way from the samples $f(j / 2 \omega)$, i.e.

$$
f(x)=\sum_{j \in \mathbb{Z}} f\left(\frac{j}{2 \omega}\right) \frac{\sin (2 \pi \omega(x-j / 2 \omega))}{2 \pi \omega(x-j / 2 \omega)},
$$

where convergence is understood in the $L_{2}$-sense. Formula (1.1) involves regularly spaced points $j / 2 \omega, j \in \mathbb{Z}$. If one would like to consider irregular sampling at a

Received by the editors August 18, 2006 and, in revised form, March 12, 2007.

2000 Mathematics Subject Classification. Primary 42C99, 05C99, 94A20; Secondary 94A12.

Key words and phrases. Combinatorial graph, combinatorial Laplace operator, discrete PaleyWiener spaces, Shannon sampling, discrete Plancherel-Polya and Poincare inequalities.

(C)2008 American Mathematical Society Reverts to public domain 28 years from publication 
sequence of points $\left\{x_{j}\right\}$ and still have a stable reconstruction from the samples $f\left(x_{j}\right)$, then the following Plancherel-Polya inequality [33, 34, should hold true:

$$
C_{1}\left(\sum_{j \in \mathbb{Z}}\left|f\left(x_{j}\right)\right|^{2}\right)^{1 / 2} \leq\|f\|_{L_{2}(\mathbb{R})} \leq C_{2}\left(\sum_{j \in \mathbb{Z}}\left|f\left(x_{j}\right)\right|^{2}\right)^{1 / 2}
$$

There is a classical result of Duffin and Schaeffer [6], where the inequalities (1.2) imply existence of a dual frame $\left\{\Theta_{j}\right\}$ which consists of functions in $P W_{\omega}(\mathbb{R})$ such that any function $f \in P W_{\omega}(\mathbb{R})$ can be reconstructed according to the following formula:

$$
f(x)=\sum_{j \in \mathbb{Z}} f\left(x_{j}\right) \Theta_{j}(x) .
$$

A similar approach can be developed for the Paley-Wiener spaces $P W_{\omega}\left(\mathbb{R}^{d}\right)$. Formula (1.3) is a generalization of formula (1.1) because it can be used for nonuniformly spaced sets of sampling points.

The theory of irregular sampling was very active for many years [1, 2], 6 , [19, 24], 23], 20], 39. Some of the ideas and methods of this theory were recently extended to the cases of Riemannian manifolds, symmetric spaces, groups, and quantum graphs [7], 8], 10, 11], 14, [15, 25]-32].

In the present article the Paley-Wiener spaces are introduced on combinatorial graphs and a corresponding sampling theory is developed which resembles the classical one. Namely it is shown that Paley-Wiener functions of low type are uniquely determined by their values on certain subgraphs (uniqueness sets) and can be reconstructed from such sets in a stable way. A description and examples of some uniqueness sets are given. A reconstruction method is presented which gives a formula of type (1.3) in terms of dual frames. More detailed consideration is given to particularly interesting cases of $n$-dimensional lattice $\mathbb{Z}^{n}$, homogeneous trees and finite graphs. Applications to eigenvalue and eigenfunction problems on finite graphs are also considered.

It seems that our results can find different applications in signal analysis, imaging, learning theory and discrete tomography [18, [36], 37].

We know just three papers [13, [16, [21] in which authors consider sampling on $\mathbb{Z}^{n}$ and on $\mathbb{Z}_{N}$, but our approach to the problem and our results are very different from the methods of these papers.

The following is a summary of the main notions and results. We consider finite or infinite, and in this case countable connected graphs $G=(V(G), E(G))$, where $V(G)$ is its set of vertices and $E(G)$ is its set of edges. We consider only simple (no loops, no multiple edges) undirected unweighted graphs. A number of vertices adjacent to a vertex $v$ is called the degree of $v$ and is denoted by $d(v)$. We assume that degrees of all vertices are bounded from above, and we use the notation

$$
d(G)=\sup _{v \in V(G)} d(v) .
$$

The space $L_{2}(G)$ is the Hilbert space of all complex-valued functions $f: V(G) \rightarrow \mathbb{C}$ with the following inner product

$$
\langle f, g\rangle=\sum_{v \in V(G)} f(v) \overline{g(v)}
$$


and the following norm

$$
\|f\|=\|f\|_{0}=\left(\sum_{v \in V(G)}|f(v)|^{2}\right)^{1 / 2} .
$$

The discrete Laplace operator $\mathcal{L}$ is defined by the formula [4]

$$
\mathcal{L} f(v)=\frac{1}{\sqrt{d(v)}} \sum_{v \sim u}\left(\frac{f(v)}{\sqrt{d(v)}}-\frac{f(u)}{\sqrt{d(u)}}\right), f \in L_{2}(G),
$$

where $v \sim u$ means that $v, u \in V(G)$ are connected by an edge. It is known that the Laplace operator $\mathcal{L}$ is a bounded operator in $L_{2}(G)$ which is self-adjoint and positive definite. Let $\sigma(\mathcal{L})$ be the spectrum of a self-adjoint positive definite operator $\mathcal{L}$ in $L_{2}(G)$. In what follows we will use the notation

$$
\omega_{\min }=\inf _{\omega \in \sigma(\mathcal{L})} \omega, \omega_{\max }=\sup _{\omega \in \sigma(\mathcal{L})} \omega .
$$

According to the spectral theory 3 there exist a direct integral of Hilbert spaces $X=\int X(\tau) d m(\tau)$ and a unitary operator $F$ from $L_{2}(G)$ onto $X$, which transforms the domain of $\mathcal{L}^{s}, s \geq 0$, onto $X_{s}=\left\{x \in X \mid \tau^{s} x \in X\right\}$ with norm

$$
\|x(\tau)\|_{X_{s}}=\left(\int_{\sigma(\mathcal{L})} \tau^{2 s}\|x(\tau)\|_{X(\tau)}^{2} d m(\tau)\right)^{1 / 2}
$$

and $F\left(\mathcal{L}^{s} f\right)=\tau^{s}(F f)$. We introduce the following notion of discrete Paley-Wiener spaces.

Definition 1. Given an $\omega \geq 0$ we will say that a function $f$ from $L_{2}(G)$ belongs to the Paley-Wiener space $P W_{\omega}(G)$ if its "Fourier transform" $F f$ has support in $[0, \omega]$.

Remark 1. To be more consistent with the definition of the classical Paley-Wiener spaces we should consider the interval $\left[0, \omega^{2}\right]$ instead of $[0, \omega]$. We prefer our choice because it makes formulas and notation simpler.

Since the operator $\mathcal{L}$ is bounded, every function from $L_{2}(G)$ belongs to a certain Paley-Wiener space $P W_{\omega}(G)$ for some $\omega \in \sigma(\mathcal{L})$, and we have the following stratification:

$$
L_{2}(G)=P W_{\omega_{\max }}(G)=\bigcup_{\omega \in \sigma(\mathcal{L})} P W_{\omega}(G), P W_{\omega_{1}}(G) \subseteq P W_{\omega_{2}}(G), \omega_{1}<\omega_{2} .
$$

Different properties of the spaces $P W_{\omega}(G)$ and in particular a generalization of the Paley-Wiener Theorem are collected in Theorem 2.1.

For a subset $S \subset V(G)$ (finite or infinite) the notation $L_{2}(S)$ will denote the space of all functions from $L_{2}(G)$ with support in $S$ :

$$
L_{2}(S)=\left\{\varphi \in L_{2}(G), \varphi(v)=0, v \in V(G) \backslash S\right\} .
$$

The following result shows that on some infinite graphs a difference between two Paley-Wiener spaces can be recognized only at infinity.

Theorem 1.1. If a graph $G$ is an n-dimensional lattice or a homogeneous tree and $\mathcal{L}$ is the Laplace operator, then for any finite set of vertices $S \subset V(G)$, any $\varphi \in L_{2}(S)$ and any $\omega \in \sigma(\mathcal{L})$ there exists a function $f_{\varphi} \in P W_{\omega}(G)$ which coincides with $\varphi$ on $S$. 
Note that the important part of this theorem that it is true for any $\omega \in \sigma(\mathcal{L})$.

Definition 2. We say that a set of vertices $U \subset V(G)$ is a uniqueness set for a space $P W_{\omega}(G), \omega>0$, if for any two functions from $P W_{\omega}(G)$, the fact that they coincide on $U$ implies that they coincide on $V(G)$.

Definition 3. We say that a set of vertices $S \subset V(G)$ is a $\Lambda$-set if for any $\varphi \in L_{2}(S)$ it admits a Poincare inequality with a constant $\Lambda>0$,

$$
\|\varphi\| \leq \Lambda\|\mathcal{L} \varphi\|, \varphi \in L_{2}(S) .
$$

The infimum of all $\Lambda>0$ for which $S$ is a $\Lambda$-set will be called the Poincare constant of the set $S$ and will be denoted by $\Lambda(S)$.

In Theorem 3.4 we give several estimates of the constant $\Lambda$ for finite sets. The role of $\Lambda$-sets is explained in the following theorem which is one of the main observations made in this paper.

Theorem 1.2. If a set $S \subset V(G)$ is a $\Lambda$-set, then the set $U=V(G) \backslash S$ is a uniqueness set for any space $P W_{\omega}(G)$ with $0<\omega<1 / \Lambda$.

Since $L_{2}(G)=P W_{\omega_{\max }}(G)$ one cannot expect that in non-trivial uniqueness sets there exist functions from every Paley-Wiener subspace $P W_{\omega}(G)$ with any $\omega_{\min } \leq \omega \leq \omega_{\max }$. Indeed, otherwise it would mean that certain subsets of vertices can be removed from a graph without changing spectral properties of $\mathcal{L}$. But it is reasonable to expect that uniqueness sets exist for Paley-Wiener spaces $P W_{\omega}(G)$ with relatively small $\omega>0$.

It is shown in this article that for every graph $G$ there exists a constant $1<$ $\Omega_{G}<\omega_{\max }$ such that for $0<\omega<\Omega_{G}$ functions from $P W_{\omega}(G)$ can be determined by using their values only on certain subsets of vertices. For instance, one can show that when $S$ is a vertex $v$ in a regular graph $G$, then the constant $\Lambda$ in (1.7) equals

$$
\Lambda(v)=\sqrt{\frac{d(v)}{d(v)+1}} .
$$

According to Theorem 1.2 it shows that any graph $G$ spaces $P W_{\omega}(G)$ with

$$
0<\omega<\sqrt{1+\frac{1}{d_{\max }}}=\Omega_{G}>1
$$

have non-trivial uniqueness sets.

We have to emphasize that our results are not trivial only for graphs for which the interval $\left[0, \Omega_{G}\right]$ has a non-empty intersection with the spectrum $\sigma(\mathcal{L})$. Below are some examples for which this condition is satisfied:

1) $n$-dimensional lattices $\mathbb{Z}^{n}$ for which the spectrum $\sigma(\mathcal{L})$ is the entire interval $[0,2]$ and $\Omega_{G}=\sqrt{1+2^{-n}}>1$.

2) Infinite countable graphs with bounded vertex degrees which have polynomial growth. For such graphs $\omega_{\min }=\inf \sigma(\mathcal{L})$ is always zero 22 .

3) Homogeneous trees of order $q+1$ for which

$$
\sigma(\mathcal{L})=[1-\eta(q), 1+\eta(q)], \Omega_{G}=\sqrt{1+(q+1)^{-1}}>1, \eta(q)=\frac{2 \sqrt{q}}{q+1} .
$$

4) Finite and not complete graphs $G$ for all of which the first non-zero eigenvalue $\lambda_{G}$ satisfies $\lambda_{G} \leq 1<\Omega_{G}$. For example for any planar graph with $n$ vertices the 
following estimate is known 22]:

$$
\lambda_{G} \leq \frac{12 d_{\max }}{\sqrt{n / 2}-6},
$$

which shows that for a fixed $d_{\max }$ and large $n$ the eigenvalue $\lambda_{G}$ is close to zero.

Given a proper set of vertices $S \subset V(G)$ its vertex boundary $b S$ is the set of all vertices in $V(G)$ which are not in $S$ but adjacent to a vertex in $S$ :

$$
b S=\{v \in V(G) \backslash S: \exists\{u, v\} \in E(G), u \in S\} .
$$

If a graph $G=(V(G), E(G))$ is connected and $S$ is a proper subset of $V$, then the vertex boundary $b S$ is not empty. For a finite set $S$ consider the set $S \cup b S=\bar{S}$ as an induced graph. It means that the graph $\bar{S}$ is determined by all edges of $G$ with both endpoints in $\bar{S}=S \cup b S$.

Definition 4. The notation $\Gamma(S)$ will be used for a graph constructed in the following way. Take two copies of the induced graph $\bar{S}=S \cup b S$, which we will denote as $\bar{S}_{1}$ and $\bar{S}_{2}$, and identify every vertex $v \in b S \subset \bar{S}_{1}$ with "the same" vertex $v \in b S \subset \bar{S}_{2}$.

The following statement summarizes some of our main results (Theorem 3.7 and Theorem 4.1).

Theorem 1.3. For a given $\omega_{\min }<\omega<\Omega_{G}$ consider a set of vertices $S=\bigcup S_{j}$ with the following properties:

1) for every $S_{j} \subset V(G)$ the following inequality takes place:

$$
\frac{1}{\lambda_{1}\left(\Gamma\left(S_{j}\right)\right)}<1 / \omega
$$

where $\lambda_{1}\left(\Gamma\left(S_{j}\right)\right)$ is the first positive eigenvalue of the graph $\Gamma\left(S_{j}\right)$;

2) the sets $\bar{S}_{j}=S_{j} \cup b S_{j}$ are disjoint.

Then the following holds true:

3) the set $U=V(G) \backslash S$ is a uniqueness set for the space $P W_{\omega}(G)$;

4) there exists a frame of functions $\left\{\Theta_{u}\right\}_{u \in U}$ in the space $P W_{\omega}(G)$ such that the following reconstruction formula holds true for all $f \in P W_{\omega}(G)$ :

$$
f(v)=\sum_{u \in U} f(u) \Theta_{u}(v), v \in V(G) .
$$

Note that the last formula is an analog of formula (1.3).

Let us illustrate some of our main results in the case of a line graph $\mathbb{Z}$. In this case the spectrum of the Laplace operator is the interval $[0,2]$. Note that since the constant $\Omega_{\mathbb{Z}}$ which is defined in (1.9) equals $\sqrt{3 / 2}$, the non-trivial uniqueness sets do not exist for spaces $P W_{\omega}(G)$ with $\omega>\sqrt{3 / 2}$.

Theorem 1.4. For any finite subset $S$ of successive vertices of the graph $\mathbb{Z}$ the following inequality holds true:

$$
\|\varphi\| \leq \frac{1}{2} \sin ^{-2} \frac{\pi}{2|S|+2}\|\mathcal{L} \varphi\|, \varphi \in L_{2}(S) .
$$

It implies that for a given $0<\omega<\sqrt{3 / 2}$ every function $f \in P W_{\omega}(G)$ is uniquely determined by its values on a set $U=V(G) \backslash S$, where $S$ is a finite or infinite union 
of disjoint sets $\left\{S_{j}\right\}$ of successive vertices such that

1) the sets $\bar{S}_{j}=S_{j} \cup b S_{j}$ are disjoint

and

2)

$$
\left|S_{j}\right|<\frac{\pi}{2 \arcsin \sqrt{\frac{\omega}{2}}}-1, j \in \mathbb{N}
$$

Moreover, there exists a frame of functions $\left\{\Theta_{u}\right\}_{u \in U}$ in the space $P W_{\omega}(G)$ such that the following reconstruction formula holds true for all $f \in P W_{\omega}(G)$ :

$$
f(v)=\sum_{u \in U} f(u) \Theta_{u}(v), v \in V(G)
$$

When $\omega$ is really small we obtain the following "Nyquist rate" of sampling:

$$
\left|S_{j}\right| \sim \frac{\pi}{\sqrt{2 \omega}}-1 \sim \frac{\pi}{\sqrt{2 \omega}}
$$

Using this theorem it is easy to estimate that if, for example, $\omega=0.5$, then there are uniqueness sets for $P W_{0.5}(\mathbb{Z})$ that contain about 50 percent of points of any interval of length $4 k$ in $\mathbb{Z}$, if $\omega=0.1$, then there are uniqueness sets for $P W_{0.1}(\mathbb{Z})$ that contain about 25 percent of points of any interval of length $8 k$ in $\mathbb{Z}$, and if $\omega=0.01$, then there are uniqueness sets for $P W_{0.01}(\mathbb{Z})$ that contain about 9 percent of points of any interval in $\mathbb{Z}$.

Remark 2. If one compares our relations (1.13) between $S_{j}$ and $\omega$ with the corresponding relations between a rate of sampling and frequency $\omega$ for classical PaleyWiener spaces, he will be confused with the presence of a square root for $\omega$. It is because there is a certain discrepancy between our definitions and classical definitions of Paley-Wiener spaces (see Remark 1).

Remark 3. Our inequalities (1.11) are similar to the inequalities which were obtained in [9]. Note that the proofs in [9] rely on the knowledge of eigenvalues of certain Hermitian matrices which were calculated by a physicist D. E. Rutherford in 35 , in connection with some problems in physics and chemistry. What is really interesting that D. E. Rutherford considered graphs as models of some physical systems.

A similar result holds true for a lattice $\mathbb{Z}^{n}$ of any dimension. The spectrum of the Laplace operator is $[0,2]$ and $\Omega_{\mathbb{Z}^{n}}=\sqrt{(2 n+1) / 2 n}$. We have the following sampling theorem.

Theorem 1.5. For a given $0<\omega<\sqrt{(2 n+1) / 2 n}$ every function $f \in P W_{\omega}(G)$ is uniquely determined by its values on a set $U=V(G) \backslash S$, where $S$ is a finite or an infinite union of disjoint $N_{1, j} \times N_{2, j} \times \ldots \times N_{n, j}$ "rectangular solids" $\left\{S_{j}\right\}$ of vertices such that

1) the sets $\bar{S}_{j}=S_{j} \cup b S_{j}$ are disjoint

and

2) the following inequality holds true for all $j$ :

$$
\omega<4 \min \left(\sin \frac{\pi}{2 N_{1, j}+2}, \sin \frac{\pi}{2 N_{2, j}+2}, \ldots, \sin \frac{\pi}{2 N_{n, j}+2}\right)=C_{j} .
$$


Moreover, there exists a frame of functions $\left\{\Theta_{u}\right\}_{u \in U}$ in the space $P W_{\omega}(G)$ such that the following reconstruction formula holds true for all $f \in P W_{\omega}(G)$ :

$$
f(v)=\sum_{u \in U} f(u) \Theta_{u}(v), v \in V(G) .
$$

We also consider Paley-Wiener spaces on homogeneous trees, and in Chapter 6 give some applications to eigenvalue and eigenfunction approximations on finite graphs. Bellow we formulate some consequences of our results about finite graphs.

Thus we assume that a graph $G$ has $N$ vertices and that eigenvalues of the Laplace operator $\mathcal{L}$ are $0=\lambda_{0}<\lambda_{1} \leq \lambda_{2} \leq \ldots \leq \lambda_{N-1}$. Let $\mathcal{N}[0, \omega)$ denote the number of eigenvalues of $\mathcal{L}$ in $[0, \omega)$ and $\mathcal{N}\left[\omega, \omega_{\max }\right]$ be a number of eigenvalues of $\mathcal{L}$ in $\left[\omega, \omega_{\max }\right]$. The notation $\mathcal{P}(\Lambda)$ is used for all sets of vertices $S \subset V(G)$ which satisfy (1.7). The next corollary gives certain information about the distribution of eigenvalues of $\mathcal{L}$.

Corollary 1.1. For any set $S$ which satisfies (1.7) the following inequalities hold true:

$$
\mathcal{N}[0,1 / \Lambda) \leq|V(G)|-|S|
$$

and

$$
\mathcal{N}\left[1 / \Lambda, \omega_{\max }\right] \geq|S| .
$$

In particular if $M=\max _{S \in \mathcal{P}(\Lambda)}|S|$, then

$$
\mathcal{N}[0,1 / \Lambda) \leq|V(G)|-M .
$$

To illustrate this result let us consider a cycle graph $C_{100}=\{1,2, \ldots, 100\}$ on 100 vertices and suppose we are going to determine all eigenvalues which are not greater than $\omega=0.002$. Note that the space $P W_{0.002}\left(C_{100}\right)$ is the span of all eigenfunctions whose eigenvalues are not greater than 0.002. According to Theorem 1.3 a uniqueness set for the space $P W_{0.002}\left(C_{100}\right)$ can be constructed as a complement of a set $S=\bigcup_{j} S_{j}$ such that $\bar{S}_{j}=S_{j} \cup b S_{j}$ are disjoint and

$$
\left|S_{j}\right|<\frac{\pi}{2 \arcsin \sqrt{\frac{0.002}{2}}}-1>49-1=48 .
$$

Thus we can take $\left|S_{j}\right|=48$, and it means that one of possible uniqueness sets $U$ will contain four vertices with numbers $1,2,51$, and 52 . According to Corollary 1.1 we can conclude that there are at most four eigenvalues of the Laplace operator which are not greater than 0.002. In fact there are three such eigenvalues $\lambda_{0}=0$, and a double eigenvalue $\lambda_{1}=1-\cos (2 \pi / 100) \approx 0.001973$.

Similar calculations show that in the case when $\omega=0.008$ the dimension of a uniqueness set $U$ can be taken equal to eight and there are five eigenvalues which are less than 0.008: $\lambda_{0}=0$ and two double eigenvalues $\lambda_{1} \approx 0.001973$, and $\lambda_{2}=$ $1-\cos (4 \pi / 100) \approx 0.007885$.

The following corollary gives a lower bound for each non-zero eigenvalue.

Corollary 1.2. If $S=\left\{v_{1}, \ldots, v_{N-k}\right\}$ is a set of $N-k$ vertices and $\Lambda(S)=$ $\Lambda\left(v_{1}, \ldots, v_{N-k}\right)$ is the corresponding Poincare constant, then the following inequality holds true:

$$
\lambda_{k} \geq \frac{1}{\Lambda\left(v_{1}, \ldots, v_{N-k}\right)}
$$


More precisely, if

$$
\Lambda_{N-k}=\min _{\left(v_{1}, \ldots, v_{N-k}\right) \in V(G)} \Lambda\left(v_{1}, \ldots, v_{N-k}\right),
$$

then

$$
\lambda_{k} \geq \frac{1}{\Lambda_{N-k}}
$$

Note that this result is "local" in the sense that any randomly chosen set $S=$ $\left\{v_{1}, \ldots, v_{N-k}\right\} \subset V(G)$ can be used to obtain an estimate of the form (1.14).

\section{Paley-Wiener spaces on COMbinatorial graphs}

The Paley-Wiener spaces $P W_{\omega}(G), \omega>0$, were introduced in Definition 1 of the Introduction. Since the operator $\mathcal{L}$ is bounded, it is clear that every function from $L_{2}(G)$ belongs to a certain Paley-Wiener space. If a graph $G$ is finite, then the space $P W_{\omega}(G)$ is a span of eigenfunctions whose eigenvalues $\leq \omega$. Note that if

$$
\omega_{\min }=\inf _{\omega \in \sigma(\mathcal{L})} \omega
$$

then the space $P W_{\omega}(G)$ is not trivial if and only if $\omega \geq \omega_{\min }$.

Using the spectral resolution of identity $P_{\lambda}$ we define the unitary group of operators by the formula

$$
e^{i t \mathcal{L}} f=\int_{\sigma(\mathcal{L})} e^{i t \tau} d P_{\tau} f, f \in L_{2}(G), t \in \mathbb{R}
$$

The next theorem can be considered as a form of the Paley-Wiener theorem, and it follows from a more general result in [27].

Theorem 2.1. The following statements hold true:

1) $f \in P W_{\omega}(G)$ if and only if for all $s \in \mathbb{R}_{+}$the following Bernstein inequality holds:

$$
\left\|\mathcal{L}^{s} f\right\| \leq \omega^{s}\|f\|
$$

2) the norm of the operator $\mathcal{L}$ in the space $P W_{\omega}(G)$ is exactly $\omega$;

3) $f \in P W_{\omega}(G)$ if and only if the following holds true:

$$
\lim _{s \rightarrow \infty}\left\|\mathcal{L}^{s} f\right\|^{1 / s}=\omega, s \in \mathbb{R}_{+} ;
$$

4) $f \in P W_{\omega}(G)$ if and only if for every $g \in L_{2}(G)$ the scalar-valued function of the real variable $t \in \mathbb{R}^{1}$,

$$
\left\langle e^{i t \mathcal{L}} f, g\right\rangle=\sum_{v \in V} e^{i t \mathcal{L}} f(v) \overline{g(v)} d(v),
$$

is bounded on the real line and has an extension to the complex plane as an entire function of the exponential type $\omega$;

5) $f \in P W_{\omega}(G)$ if and only if the abstract-valued function $e^{i t \mathcal{L}} f$ is bounded on the real line and has an extension to the complex plane as an entire function of the exponential type $\omega$;

6) $f \in P W_{\omega}(G)$ if and only if the solution $u(t, v), t \in \mathbb{R}^{1}, v \in V(G)$, of the Cauchy problem for the corresponding Schrodinger equation

$$
i \frac{\partial u(t, v)}{\partial t}=\mathcal{L} u(t, v), u(0, v)=f(v), i=\sqrt{-1},
$$


has an analytic extension $u(z, v)$ to the complex plane $\mathbb{C}$ as an entire function and satisfies the estimate

$$
\|u(z, \cdot)\|_{L_{2}(G)} \leq e^{\omega|\Im z|}\|f\|_{L_{2}(G)} .
$$

This theorem gives the following stratification:

$$
L_{2}(G)=P W_{\omega_{\max }}(G)=\bigcup_{\omega_{\min } \leq \omega \leq \omega_{\max }} P W_{\omega}(G), P W_{\omega}(G) \subseteq P W_{\sigma}(G), \omega<\sigma,
$$

of the space of all $L_{2}(G)$-functions. The theorem shows that the notion of PaleyWiener functions of type $\omega$ on a combinatorial graph can be completely understood in terms of familiar entire functions of exponential type $\omega$ bounded on the real line.

\section{Uniqueness Sets for discrete Paley-Wiener functions And Plancherel-Polya and Poincare inequalities on GraphS}

The definition of uniqueness sets was given in the Introduction. The following theorem gives necessary and sufficient conditions for being a uniqueness set.

Theorem 3.1. A set of vertices $U \subset V(G)$ is a uniqueness set for the space $P W_{\omega}(G)$ if and only if there exists a constant $C_{\omega}$ such that for any $f \in P W_{\omega}(G)$ the following discrete version of the Plancherel-Polya inequalities holds true:

$$
\left(\sum_{u \in U}|f(u)|^{2}\right)^{1 / 2} \leq\|f\|_{L_{2}(G)} \leq C_{\omega}\left(\sum_{u \in U}|f(u)|^{2}\right)^{1 / 2}
$$

for all $f \in P W_{\omega}(G)$.

Proof. The closed linear subspace $P W_{\omega}(G)$ is a Hilbert space with respect to the norm of $L_{2}(G)$. At the same time since $U \subset V(G)$ is a uniqueness set for $P W_{\omega}(G)$ the functional

$$
|||f|||=\left(\sum_{u \in U}|f(u)|^{2}\right)^{1 / 2}
$$

defines another norm on $P W_{\omega}(G)$. Indeed, the only property which should be verified is that the condition $\left|\|f \mid\|=0, f \in P W_{\omega}(G)\right.$, implies that $f$ is identical to zero on the entire graph, but it is guaranteed by the fact that $U$ is a uniqueness set for $P W_{\omega}(G)$.

Since for any $f \in P W_{\omega}(G)$ the norm $\||f|\|$ is not greater than the original norm $\|f\|_{L_{2}(G)}$, the closed graph theorem implies the existence of a constant $C_{\omega}$ for which the reverse inequality holds true.

We will also need the following corollary which is easy to prove.

Corollary 3.1. In the same notation as above if $\mathcal{B}$ is an operator in $L_{2}(G)$ such that its restriction to $P W_{\omega}(G)$ is a bounded invertible operator from $P W_{\omega}(G)$ onto $P W_{\omega}(G)$, then

$$
\begin{gathered}
\frac{1}{\|\mathcal{B}\|}\left(\sum_{u \in U}|\mathcal{B} f(u)|^{2}\right)^{1 / 2} \\
\leq\|f\|_{L_{2}(G)} \leq\left\|\mathcal{B}^{-1}\right\| C_{\omega}\left(\sum_{u \in U}|\mathcal{B} f(u)|^{2}\right)^{1 / 2}
\end{gathered}
$$

for all $f \in P W_{\omega}(G)$. 
Remark 4. It is worth noting that the statement similar to Theorem 3.1 does not hold true for Paley-Wiener spaces on $\mathbb{R}^{d}$. Namely, not every uniqueness set is associated with a corresponding Plancherel-Polya inequality. Sets of points $\left\{x_{j}\right\} \in$ $\mathbb{R}^{d}$ for which a Plancherel-Polya inequality takes place along with a separation condition $\left|x_{j}-x_{k}\right|>\delta>0, j \neq k$, are known as sampling sets.

For a general graph $G$ an important example of the operator $\mathcal{B}$ from the corollary is the operator $(\varepsilon I+\mathcal{L})^{s}$ for any positive $\varepsilon>0$ and any real $s \in \mathbb{R}$.

Our next goal is to develop some sufficient conditions on a set of vertices for being a uniqueness set for a Paley-Wiener subspace. It turns out that it is easier to understand complements of uniqueness sets for spaces $P W_{\omega}(G)$.

We now turn to the notion of a $\Lambda$-set which was introduced in Definition 3 in the Introduction. Let us consider one of our main examples of $\Lambda$-sets.

Example 1. Let $v \in V$ be any vertex in a connected graph $G(V(G), E(G))$. In this case the corresponding space $L_{2}(\{v\})$ consists of all functions proportional to the Dirac measure $\delta_{v}$. Simple calculations show

$$
\mathcal{L} f(v)=1, \mathcal{L} f(w)=-\frac{1}{\sqrt{d(w) d(v)}}, w \sim v
$$

and $\mathcal{L} f(u)=0$ for all other vertices $u \in V(G)$. Thus we have

$$
\left\|\delta_{v}\right\|=\Lambda(v)\left\|\mathcal{L} \delta_{v}\right\|
$$

where $\Lambda(v)$ is

$$
\frac{1}{\sqrt{2}}<\Lambda(v)=\frac{1}{\sqrt{1+\frac{1}{d(v)} \sum_{w \sim v} \frac{1}{d(w)}}}<1, d(v) \geq 1 .
$$

This example can be used to construct examples of infinite $\Lambda$-sets. For example, we can consider an infinite path on a rectangular grid or on a tree. These situations will be generalized in Lemma 3.6.

The role of $\Lambda$-sets is explained in the following theorem.

Theorem 3.2. If a set $S \subset V(G)$ is a $\Lambda$-set, then $S$ is $P W_{\omega}(G)$-removable for any $\omega<1 / \Lambda$, i.e. the set $U=V(G) \backslash S$ is a uniqueness set for any space $P W_{\omega}(G)$ with $\omega<1 / \Lambda$.

Proof. If $f, g \in P W_{\omega}(G)$, then $f-g \in P W_{\omega}(G)$, and according to Theorem 2.1 the following Bernstein inequality holds true:

$$
\|\mathcal{L}(f-g)\| \leq \omega\|f-g\| .
$$

If $f$ and $g$ coincide on $U=V(G) \backslash S$, then $f-g$ belongs to $L_{2}(S)$, and since $S$ is a $\Lambda$-set it should satisfy

$$
\|\varphi\| \leq \Lambda\|\mathcal{L} \varphi\|, \varphi \in L_{2}(S) .
$$

Thus, if $f-g$ is not zero and $\omega<1 / \Lambda$, we have the following inequalities:

$$
\|f-g\| \leq \Lambda\|\mathcal{L}(f-g)\| \leq \Lambda \omega\|f-g\|<\|f-g\|,
$$

which contradict the assumption that $f-g$ is not identical zero. It proves the theorem. 
This theorem and the estimate (3.5) show that for any graph $G$ spaces $P W_{\omega}(G)$ with

$$
0<\omega<\sqrt{\frac{1+d(G)}{d(G)}}=\Omega_{G}>1, d(G)=\sup _{v \in V(G)} d(v),
$$

have non-trivial uniqueness sets.

Now we show that every finite set admits a Poincare inequality.

Theorem 3.3. Assume that $S$ is a finite set with non-empty boundary. If $\mathcal{L}_{\Gamma(S)}$ is the Laplacian on the graph $\Gamma(S)$ and $\lambda_{1}(\Gamma(S))$ is its smallest non-zero eigenvalue, then for every $\varphi \in L_{2}(S)$ the following inequality holds true:

$$
\|\varphi\|_{L_{2}(G)} \leq \frac{1}{\lambda_{1}(\Gamma(S))}\left\|\mathcal{L}_{G} \varphi\right\|_{L_{2}(G)}
$$

Proof. Let us recall the construction of the graph $\Gamma(S)$. We consider $\bar{S}=S \cup b S$ as an induced graph and take two copies of it, which we will denote as $\bar{S}_{1}$ and $\bar{S}_{2}$. We identify every vertex $v \in b S \subset \bar{S}_{1}$ with "the same" vertex $v \in b S \subset \bar{S}_{2}$. We construct an embedding of the space $L_{2}(S)$ into the space $L_{2}(\Gamma(S))$. If $\varphi \in L_{2}(S)$, then its image $F_{\varphi} \in L_{2}(\Gamma(S))$ is defined according to the following rules:

1) $F_{\varphi}(v)=\varphi(v)$, for every $v \in \bar{S}_{1}$,

2) $F_{\varphi}(v)=-\varphi(v)$, for every $v \in \bar{S}_{2}$.

As direct calculations show, the following holds true for every function $\varphi \in$ $L_{2}(S)$ :

$$
\left\|F_{\varphi}\right\|_{L_{2}(\Gamma(S))}=2\|\varphi\|_{L_{2}(G)} .
$$

We will use notation $d_{G}(v), d_{\bar{S}}(v)$, and $d_{\Gamma(S)}(v)$ for degrees of a vertex $v$ in $\bar{S}$ considered as a vertex of $G$, or as a vertex of the induced graph $\bar{S}$ or as a vertex of the new graph $\Gamma(S)$, respectively. It is clear that $d_{\Gamma(S)}(v) \leq d_{\bar{S}}(v)+1$ and $d_{\bar{S}}(v) \leq d_{G}(v)$ for every $v \in \bar{S}$. Thus in general $d_{\Gamma(S)}(v) \leq d_{G}(v)+1 \leq 2 d_{G}(v)$, and it results in the following estimate:

$$
\left\|\mathcal{L}_{\Gamma(S)} F_{\varphi}\right\|_{L_{2}(\Gamma(S))} \leq 2\left\|\mathcal{L}_{G} \varphi\right\|_{L_{2}(G)} .
$$

The eigenfunction of $\mathcal{L}_{\Gamma(S)}$ that corresponds to the 0 -eigenvalue is given by the formula $\psi_{0}(v)=\sqrt{d_{\Gamma(S)}(v)}, v \in \Gamma(S)$. Since every function $F_{\varphi}$ is "odd", it is orthogonal to the subspace spanned by $\psi_{0}$. Because of it if $\left\{\psi_{j}\right\}, j=0,1, \ldots, N$, is a complete orthonormal system of eigenfunctions of $\mathcal{L}_{\Gamma(S)}$ in $L_{2}(\Gamma(S))$ and $0=$ $\lambda_{0}(\Gamma(S))<\lambda_{1}(\Gamma(S)) \leq \ldots \leq \lambda_{N}(\Gamma(S))$ is a set of their corresponding eigenvalues, the following formulas hold true:

$$
F_{\varphi}=\sum_{j=1}^{N}\left\langle F_{f}, \psi_{j}\right\rangle \psi_{j} ; \mathcal{L}_{\Gamma(S)} F_{\varphi}=\sum_{j=1}^{N} \lambda_{j}(\Gamma(S))\left\langle F_{\varphi}, \psi j\right\rangle \psi_{j}
$$

and

$$
\left\|\mathcal{L}_{\Gamma(S)} F_{\varphi}\right\|_{L_{2}(G)}^{2}=\sum_{j=1}^{N} \lambda_{j}^{2}(\Gamma(S))\left|\left\langle F_{\varphi}, \psi_{j}\right\rangle\right|^{2} \geq \lambda_{1}^{2}(\Gamma(S))\left\|F_{\varphi}\right\|_{L_{2}(G)}^{2} .
$$

This inequality along with (3.8) and (3.9) imply the theorem.

We are going to make use of some known estimates [4] on the first eigenvalue of a finite graph. A graph $\Gamma$ has isoperimetric dimension $\delta$ with isoperimetric constant 
$c_{\delta}$ if for any subset $W$ of $V(\Gamma)$, the number of edges between $W$ and the complement $W^{\prime}$ of $W$, denoted by $|\partial W|=\left|E\left(W, W^{\prime}\right)\right|$, satisfies

$$
\left|E\left(W, W^{\prime}\right)\right| \geq c_{\delta}(\operatorname{vol} W)^{\frac{\delta-1}{\delta}} .
$$

If $\delta$ is the isoperimetric dimension of the graph $\Gamma$, then there exists a constant $C_{\delta}$ which depends just on $\delta$ such that

$$
\lambda_{1}(\Gamma)>C_{\delta}\left(\frac{1}{\operatorname{vol} \Gamma}\right)^{2 / \delta}
$$

where the volume $\operatorname{vol}(\Gamma)$ of a graph $\Gamma$ is defined as

$$
\operatorname{vol}(\Gamma)=\sum_{v \in V(\Gamma)} d(v)
$$

From the construction of the graph $\Gamma(S)$ it is clear that

$$
\operatorname{vol}(\Gamma(S))=2\left(\operatorname{vol} S+\operatorname{vol}_{R}(b S)\right) \leq 2(\operatorname{vol} S+\operatorname{vol}(b S)),
$$

where $\left.\operatorname{vol}_{R}(b S)\right)$ means that the volume of the set $b S$ is calculated under the assumption that this set is a subset if the induced graph is $R$. There is another lower estimate of the first non-zero eigenvalue of a graph in terms of the Cheeger constant $h_{\Gamma}$. To define $h_{\Gamma}$ we define

$$
h_{\Gamma}(W)=\frac{\left|E\left(W, W^{\prime}\right)\right|}{\min \left(\operatorname{vol} W, \operatorname{vol} W^{\prime}\right)}
$$

and then

$$
h_{\Gamma}=\min _{W} h_{\Gamma}(W) .
$$

The following lower estimate is known for any connected graph $\Gamma$ :

$$
\lambda_{\Gamma}>\frac{h_{\Gamma}^{2}}{2}
$$

There is a lower estimate on the first eigenvalue in terms of a diameter and volume of a graph. Namely, the following estimate follows from the variational principal 4]:

$$
\lambda_{1}(\Gamma) \geq \frac{1}{D(\Gamma) \operatorname{vol}(\Gamma)} .
$$

This estimate along with the last theorem gives the following estimate:

$$
\|f\|_{L_{2}(G)} \leq 2 D(\Gamma(S)) \operatorname{vol}(\Gamma(S))\left\|\mathcal{L}_{G} f\right\|_{L_{2}(G)}
$$

for every function from $L_{2}(G)$ with support in $S$. The construction of the graph $\Gamma(S)$ implies the inequality

$$
D(\Gamma(S)) \leq 2 D(S)+1 .
$$

Altogether it gives the following result.

Theorem 3.4. If $S$ is a finite set of vertices of a graph $G$ and $\Gamma(S)$ is the same as above, then for any $\varphi \in L_{2}(S)$ the following Poincare-type inequalities hold true:

$$
\begin{aligned}
& \|\varphi\|_{L_{2}(G)} \leq \frac{4}{h_{\Gamma(S)}^{2}}\left\|\mathcal{L}_{G} \varphi\right\|_{L_{2}(G)}, \\
& \|\varphi\|_{L_{2}(G)} \leq 2 D(\Gamma(S)) \operatorname{vol}(\Gamma(S))\left\|\mathcal{L}_{G} \varphi\right\|_{L_{2}(G)}, \\
& \|\varphi\|_{L_{2}(G)} \leq 4(2 D(S)+1)\left(\operatorname{vol}_{S}+\operatorname{vol}_{R}(b S)\right)\left\|\mathcal{L}_{G} \varphi\right\|_{L_{2}(G)} \text {. }
\end{aligned}
$$


There exists a $C_{\delta}>0$ which depends only on the isoperimetric constant $\delta$ of the graph $\Gamma(S)$ such that

$$
\begin{gathered}
\|\varphi\|_{L_{2}(G)} \leq C_{\delta}^{-1}(\operatorname{vol} \Gamma(S))^{2 / \delta}\left\|\mathcal{L}_{G} \varphi\right\|_{L_{2}(G)}, \\
\|\varphi\|_{L_{2}(G)} \leq C_{\delta}^{-1}\left(2\left(\operatorname{vol} S+\operatorname{vol}_{R}(b S)\right)\right)^{2 / \delta}\left\|\mathcal{L}_{G} \varphi\right\|_{L_{2}(G)} .
\end{gathered}
$$

Note that since the isoperimetric dimension $\delta$ is a generalization of such a notion as the dimension of a manifold, the estimate (3.10) is a generalization of the following estimate for the classical Laplace operator $\Delta$ on a compact domain $S \subset \mathbb{R}^{d}$ :

$$
\|\varphi\|_{L_{2}\left(\mathbb{R}^{d}\right)} \leq C_{d}(\operatorname{diam} S)^{2}\|\Delta \varphi\|_{L_{2}\left(\mathbb{R}^{d}\right)}, \varphi \in C_{0}^{\infty}(S) .
$$

In the case of $\mathbb{R}^{d}$ such kind inequalities play an important role in harmonic analysis and differential equations and are usually associated with the names of Wirtinger, Poincare, and Sobolev [9], 17, [38].

The following two lemmas describe some infinite $\Lambda$-sets. The first lemma is obvious.

Lemma 3.5. Suppose that a set of vertices $S \subset V(G)$ (finite or infinite) has the property that for any $v \in S$ its closure $\bar{v}=v \cup b v$ does not contain other points of $S$. Then $S$ is a $\Lambda$-set with $\Lambda=1$, i.e.

$$
\|\varphi\| \leq\|\mathcal{L} \varphi\|, \varphi \in L_{2}(S) .
$$

Lemma 3.6. Suppose that for a set of vertices $S \subset V(G)$ (finite or infinite) the following conditions hold true:

1) every point from $S$ is adjacent to a point from the boundary;

2) for every $v \in S$ there exists at least one adjacent point $u_{v} \in b S$ whose adjacency set intersects $S$ only over $v$;

3) the number

$$
\Lambda=\sup _{v \in S} d(v)
$$

is finite. Then the set $S$ is a $\Lambda$-set.

Proof. Assumptions of the lemma imply that there exists a subset $S^{*} \subset b S$ such that for every vertex $v \in S$ there exists at least one point $u_{v} \in S^{*}$ whose adjacency set intersects $S$ only over $v$. A direct calculation shows that if $\varphi \in L_{2}(S)$, then

$$
\mathcal{L} \varphi\left(u_{v}\right)=-\frac{\varphi(v)}{\sqrt{d(v) d\left(u_{v}\right)}}, u_{v} \in S^{*}, v \in S .
$$

Define $\Lambda$ by the formula (3.12). Since by assumption for every $v \in S$ there exists at least one vertex $u_{v}$ in $S^{*}$ which is adjacent to $v$, we obtain

$$
\|\mathcal{L} \varphi\| \geq\left(\sum_{v \in S}\left|\mathcal{L} \varphi\left(u_{v}\right)\right|^{2} d\left(u_{v}\right)\right)^{1 / 2} \geq \Lambda^{-1}\|\varphi\| .
$$

In particular if degrees of all vertices in $S$ and $S^{*}$ are uniformly bounded from above by a number $d(G)$, then $\Lambda \leq d(G)$. The lemma is proved.

The following property is important and allows us to construct infinite $\Lambda$-sets from the finite ones. 
Lemma 3.7. Suppose that $\left\{S_{j}\right\}$ is a finite or an infinite sequence of disjoint subsets of vertices $S_{j} \subset V$ such that the sets $S_{j} \cup b S_{j}$ are pairwise disjoint. Then if a set $S_{j}$ has type $\Lambda_{j}$, then their union $S=\bigcup_{j} S_{j}$ is a set of type $\Lambda=\sup _{j} \Lambda_{j}$.

Proof. Since the sets $S_{j}$ are disjoint, every function $\varphi \in L_{2}(S), S=\bigcup_{j} S_{j}$, is a sum of functions $\varphi_{j} \in L_{2}\left(S_{j}\right)$ which are pairwise orthogonal. Moreover because the sets $S_{j} \cup b S_{j}$ are disjoint, the functions $\mathcal{L} \varphi_{j}$ are also orthogonal. Thus we have

$$
\|\varphi\|^{2}=\sum_{j}\left\|\varphi_{j}\right\|^{2} \leq \sum_{j} \Lambda_{j}^{2}\left\|\mathcal{L} \varphi_{j}\right\|^{2} \leq \Lambda^{2}\|\mathcal{L} \varphi\|^{2},
$$

where $\Lambda=\sup _{j} \Lambda_{j}$. The lemma is proved.

A combination of the last lemma and Theorem 3.3 gives the following uniqueness result.

Theorem 3.8. For a given $\omega_{\min }<\omega<\Omega_{G}$ consider a set $S=\bigcup_{j} S_{j}$ with the following properties:

1) for every $S_{j}$ the following inequality takes place:

$$
\frac{1}{\lambda_{1}\left(\Gamma\left(S_{j}\right)\right)}<1 / \omega
$$

2) the sets $S_{j} \cup b S_{j}$ are disjoint.

Then the set $U=V(G) \backslash S$ is a uniqueness set for the space $P W_{\omega}(G)$.

Although the space $L_{2}(S)$ is not invariant under $\mathcal{L}$ the inequality (1.7) implies infinitely many similar inequalities. Namely, we have the following result which will be used later.

Lemma 3.9. If $S$ is a $\Lambda$-set, then for any $\varphi \in L_{2}(S)$ and all $t \geq 0, k=2^{l}, l=$ $0,1,2, \ldots$,

$$
\left\|\mathcal{L}^{t} \varphi\right\| \leq \Lambda^{k}\left\|\mathcal{L}^{k+t} \varphi\right\|, \varphi \in L_{2}(S)
$$

in particular

$$
\|\varphi\| \leq \Lambda^{k}\left\|\mathcal{L}^{k} \varphi\right\|, \varphi \in L_{2}(S) .
$$

Proof. By the spectral theory [3] there exist a direct integral of Hilbert spaces $X=\int X(\tau) d m(\tau)$ and a unitary operator $F$ from $L_{2}(G)$ onto $X$, which transforms the domain of $\mathcal{L}^{t}, t \geq 0$, onto $X_{t}=\left\{x \in X \mid \tau^{t} x \in X\right\}$ with norm

$$
\left\|\mathcal{L}^{t} f\right\|_{L_{2}(G)}=\left(\int_{\mathbb{R}_{+}} \tau^{2 t}\|F f(\tau)\|_{X(\tau)}^{2} d m(\tau)\right)^{1 / 2}
$$

and $F\left(\mathcal{L}^{t} f\right)=\tau^{t}(F f)$. For any $\varphi \in L_{2}(G)$ we have

$$
\int_{\mathbb{R}_{+}}|F \varphi(\tau)|^{2} d m(\tau) \leq \Lambda^{2} \int_{\mathbb{R}_{+}} \tau^{2}|F \varphi(\tau)|^{2} d m(\tau),
$$

and then for the ball $B=B\left(0, \Lambda^{-1}\right)$ we have

$$
\begin{gathered}
\int_{B}|F \varphi(\tau)|^{2} d m(\tau)+\int_{\mathbb{R}_{+} \backslash B}|F \varphi|^{2} d m(\tau) \\
\leq \Lambda^{2}\left(\int_{B} \tau^{2}|F \varphi|^{2} d m(\tau)+\int_{\mathbb{R}_{+} \backslash B} \tau^{2}|F \varphi|^{2} d m(\tau)\right) .
\end{gathered}
$$


Since $\Lambda^{2} \tau^{2}<1$ on $B\left(0, \Lambda^{-1}\right)$

$$
0 \leq \int_{B}\left(|F \varphi|^{2}-\Lambda^{2} \tau^{2}|F \varphi|^{2}\right) d m(\tau) \leq \int_{\mathbb{R}_{+} \backslash B}\left(\Lambda^{2} \tau^{2}|F \varphi|^{2}-|F \varphi|^{2}\right) d m(\tau) .
$$

This inequality implies the inequality

$0 \leq \int_{B}\left(\Lambda^{2} \tau^{2}|F \varphi|^{2}-\Lambda^{4} \tau^{4}|F \varphi|^{2}\right) d m(\tau) \leq \int_{\mathbb{R}_{+} \backslash B}\left(\Lambda^{4} \tau^{4}|F \varphi|^{2}-\Lambda^{2} \tau^{2}|F \varphi|^{2}\right) d m(\tau)$

or

and then

$$
\Lambda^{2} \int_{\mathbb{R}_{+}} \tau^{2}|F \varphi|^{2} d m(\tau) \leq \Lambda^{4} \int_{\mathbb{R}_{+}} \tau^{4}|F \varphi|^{2} d m(\tau)
$$

$$
\|\varphi\| \leq \Lambda\|\mathcal{L} \varphi\| \leq \Lambda^{2}\left\|\mathcal{L}^{2} \varphi\right\|, \varphi \in L_{2}(S) .
$$

Next by using induction one can show the inequality $\|\varphi\| \leq \Lambda^{k}\left\|\mathcal{L}^{k} \varphi\right\|$ for any $k=2^{l}, l=0,1, \ldots$. Then again, because for any $t \geq 0, \Lambda^{2 t} \tau^{2 t}<1$ on $B\left(0, \Lambda^{-1}\right)$ we have

$$
\begin{aligned}
& 0 \leq \int_{B}\left(\Lambda^{2 t} \tau^{2 t}|F \varphi|^{2}-\Lambda^{2(k+t)} \tau^{2(k+t)}|F \varphi|^{2}\right) d m(\tau) \\
& \leq \int_{\mathbb{R}_{+} \backslash B}\left(\Lambda^{2(k+t)} \tau^{2(k+t)}|F \varphi|^{2}-\Lambda^{2 t} \tau^{2 t}|F \varphi|^{2}\right) d m(\tau),
\end{aligned}
$$

which gives $\left\|\mathcal{L}^{t} \varphi\right\| \leq \Lambda^{k}\left\|\mathcal{L}^{(k+t)} \varphi\right\|, \varphi \in L_{2}(S)$. The lemma is proved.

\section{A Reconstruction ALgorithm}

\section{IN TERMS OF DUAL FRAMES IN HILBERT SPACES}

In this section we will use Theorem 3.1 to develop a reconstruction method in terms of Hilbert frames. Recall that a set of vectors $\left\{h_{j}\right\}$ from a Hilbert space $H$ is called a frame in $H$ if there are $0<A<B$ such that for any $f \in H$

$$
A \sum_{j}\left|\left\langle f, h_{j}\right\rangle_{H}\right|^{2} \leq\|f\|_{H}^{2} \leq B \sum_{j}\left|\left\langle f, h_{j}\right\rangle_{H}\right|^{2},
$$

where $\langle., .\rangle_{H}$ is the inner product in $H$. The ratio $A / B$ is called the tightness of the frame.

Let $\delta_{v} \in L_{2}(G)$ be a Dirac measure supported at a vertex $v \in V$. The notation $\vartheta_{v}$ will be used for a function which is an orthogonal projection of the function

$$
\frac{1}{\sqrt{d(v)}} \delta_{v}
$$

on the subspace $P W_{\omega}(G)$. Then the Plancherel-Polya inequalities (3.1) can be written in the form

$$
\sum_{u \in U}\left|\left\langle f, \vartheta_{u}\right\rangle\right|^{2} \leq\|f\|_{L_{2}(G)}^{2} \leq C_{\omega}^{2} \sum_{u \in U}\left|\left\langle f, \vartheta_{u}\right\rangle\right|^{2},
$$

where $f, \vartheta_{u} \in P W_{\omega}(G)$ and $\left\langle f, \vartheta_{u}\right\rangle$ is the inner product in $L_{2}(G)$. These inequalities mean that if $U$ is a uniqueness set for the subspace $P W_{\omega}(G)$, then the functions $\left\{\vartheta_{u}\right\}_{u \in U}$ form a frame in the subspace $P W_{\omega}(G)$ and the tightness of this frame is $1 / C_{\omega}^{2}$. Following an idea of Duffin and Schaeffer [6] we sketch the proof of the following theorem which gives a reconstruction formula similar to formulas (1.1) and (1.4). 
Theorem 4.1. If $U \subset V(G)$ is a uniqueness set for the subspace $P W_{\omega}(G)$, then there exists a frame of functions $\left\{\Theta_{u}\right\}_{u \in U}$ in the space $P W_{\omega}(G)$ such that the following reconstruction formula holds true for all $f \in P W_{\omega}(G)$ :

$$
f(v)=\sum_{u \in U} f(u) \Theta_{u}(v), v \in V(G) .
$$

Proof. The idea is to show that the so-called frame operator

$$
F f=\sum_{u \in U}\left\langle f, \vartheta_{u}\right\rangle \vartheta_{u}, \quad f \in P W_{\omega}(G),
$$

is an automorphism of the space $P W_{\omega}(G)$ onto itself and $\|F\| \leq C_{\omega},\left\|F^{-1}\right\| \leq 1$. We consider an increasing sequence of finite subsets of $U$

$$
U_{1} \subset U_{2} \subset \ldots \subset U
$$

and introduce the operator $F_{j}: P W_{\omega}(G) \rightarrow P W_{\omega}(G)$, which is given by the formula

$$
F_{j} f=\sum_{u \in U_{j}}\left\langle f, \vartheta_{u}\right\rangle \vartheta_{u}, \quad f \in P W_{\omega}(G) .
$$

It can be shown that the Plancherel-Polya inequalities (4.1) imply that the limit

$$
\lim _{j \rightarrow \infty} F_{j} f=F f, \quad f \in P W_{\omega}(G),
$$

exists. We also have

$$
\|F f\|^{2}=\sup _{\|h\|=1}\left|\sum_{u \in U}\left\langle f, \vartheta_{u}\right\rangle\left\langle\vartheta_{u}, h\right\rangle\right|^{2} \leq \sup _{\|h\|=1} C_{\omega}^{2}\|f\|^{2}\|h\|^{2}=C_{\omega}^{2}\|f\|^{2},
$$

which shows that the operator $F$ is continuous. The same Plancherel-Polya inequalities (4.1) imply that $I \leq F \leq C_{\omega} I$, where $I$ is the identity operator. Thus, we have

and then

$$
0 \leq I-C_{\omega}^{-1} F \leq I-C_{\omega}^{-1} I=\left(C_{\omega}-1\right) C_{\omega}^{-1} I,
$$

$$
\left\|I-C_{\omega}^{-1} F\right\| \leq\left\|\left(C_{\omega}-1\right) C_{\omega}^{-1} I\right\| \leq\left(C_{\omega}-1\right) C_{\omega}^{-1}<1 .
$$

It shows that the operator $\left(C_{\omega}^{-1} F\right)^{-1}$ and consequently the operator $F^{-1}$ are bounded operators and the Neumann series gives the desired estimate $\left\|F^{-1}\right\| \leq 1$ :

$$
F^{-1}=C_{\omega}^{-1}\left(C_{\omega}^{-1} F\right)^{-1}=C_{\omega}^{-1} \sum_{k=0}^{\infty}\left(I-C_{\omega}^{-1} F\right)^{k} .
$$

Thus we have

$$
f=F^{-1} F f=\sum_{u \in U}\left\langle f, \vartheta_{u}\right\rangle \Theta_{u}
$$

where the functions $\Theta_{u}=F^{-1} \vartheta_{u}$ form a dual frame $\left\{\Theta_{u}\right\}_{u \in U}$ in the space $P W_{\omega}(G)$. The theorem is proved.

In a similar way we can prove the following result about a "derivative sampling".

Theorem 4.2. If $U \subset V(G)$ is a uniqueness set for the subspace $P W_{\omega}(G)$, then for any $s \in \mathbb{R}$ there exists a frame of functions $\left\{\Phi_{u}^{(s)}\right\}_{u \in U}$ in the space $P W_{\omega}(G)$ such that the following reconstruction formula holds true for all $f \in P W_{\omega}(G)$ :

$$
f(v)=\sum_{u \in U}(I+\mathcal{L})^{s} f(u) \Phi_{u}^{(s)}(v), v \in V(G) .
$$


If $U \subset V(G)$ is a uniqueness set for a space $P W_{\omega}(G)$, the notation $l_{2, \omega}(U)$ will be used for a linear subspace of all sequences $a=\left\{a_{u}\right\}, u \in U$, in $l_{2}$ for which there exists a function $f$ in $P W_{\omega}(G)$ such that

$$
f(u)=a_{u}, u \in U .
$$

In general $l_{2, \omega}(U) \neq l_{2}$. A linear reconstruction method $\mathcal{R}$ is a linear operator $\mathcal{R}: l_{2, \omega}(U) \rightarrow P W_{\omega}(G)$ such that

$$
\mathcal{R}: y \rightarrow f, y=\left\{y_{u}\right\}, y_{u}=f(u), u \in U .
$$

The reconstruction method $\mathcal{R}$ is said to be stable if it is continuous. We obviously have the following statement about stable reconstruction from derivatives.

Corollary 4.1. For any uniqueness set $U$ and any $s \in \mathbb{R}$ the reconstruction of $f$ from the corresponding set of samples $\left\{(I+\mathcal{L})^{s} f(u)\right\}, u \in U$, is stable.

\section{LAtTice $\mathbb{Z}^{n}$ AND homogeneous trees}

We consider a one-dimensional lattice $\mathbb{Z}$. The dual group of the commutative additive group $\mathbb{Z}$ is the one-dimensional torus. The corresponding Fourier transform $\mathcal{F}$ on the space $L_{2}(\mathbb{Z})$ is defined by the formula

$$
\mathcal{F}(f)(\xi)=\sum_{k \in \mathbb{Z}} f(k) e^{i k \xi}, f \in L_{2}(\mathbb{Z}), \xi \in[-\pi, \pi) .
$$

It gives a unitary operator from $L_{2}(\mathbb{Z})$ on the space $L_{2}(\mathbb{T})=L_{2}(\mathbb{T}, d \xi / 2 \pi)$, where $\mathbb{T}$ is the one-dimensional torus and $d \xi / 2 \pi$ is the normalized measure. One can verify the following formula:

$$
\mathcal{F}(\mathcal{L} f)(\xi)=2 \sin ^{2} \frac{\xi}{2} \mathcal{F}(f)(\xi)
$$

The next result is obvious.

Theorem 5.1. The spectrum of the Laplace operator $\mathcal{L}$ on the one-dimensional lattice $\mathbb{Z}$ is the set $[0,2]$. A function $f$ belongs to the space $P W_{\omega}(\mathbb{Z}), 0<\omega<2$, if and only if the support of $\mathcal{F} f$ is a subset $\Omega_{\omega}$ of $[-\pi, \pi)$ on which $2 \sin ^{2} \frac{\xi}{2} \leq \omega$.

To formulate the next theorem we introduce the restriction operator

$$
\mathcal{R}_{\omega}^{S}: P W_{\omega}(\mathbb{Z}) \rightarrow L_{2}(S), S \subset V(\mathbb{Z}),
$$

where

$$
\mathcal{R}_{\omega}^{S}(\varphi)=\left.\varphi\right|_{S}, \varphi \in P W_{\omega}(\mathbb{Z}) .
$$

Theorem 5.2. For any finite set of vertices $S \subset \mathbb{Z}$ and for every $\omega>0$ the restriction operator $\mathcal{R}_{\omega}^{S}$ is surjective.

Proof. Assume that a function $h \in L_{2}(S)$ is orthogonal to restrictions of all functions from a space $P W_{\omega}(\mathbb{Z}), 0<\omega<2$, to a finite $S$. Since $h$ has support on a finite set $S$ its Fourier transform $\mathcal{F}$ is a finite combination of exponents and in particular an analytic function on the real line. It implies that the set of zeros of $\mathcal{F} h$ has measure zero. At the same time by Parseval's relation this function should be orthogonal to any function with support in the set $\Omega_{\omega}$ which is a set of positive measure. This contradiction shows that there is no function in $L_{2}(S)$ which is orthogonal to restrictions to $S$ of all functions from $P W_{\omega}(\mathbb{Z})$. Since the set $S$ is finite, the space $L_{2}(S)$ is finite dimensional and implies that $L_{2}(S)$ is exactly the set of all restrictions of $P W_{\omega}(\mathbb{Z})$ to $S$. The theorem is proved. 
Our nearest goal is to show that for a one-dimensional line graph $\mathbb{Z}$ the estimates in Poincare inequalities of finite sets can be improved and all the constants can be computed explicitly. What follows is a specific realization of the construction and of the proof of Theorem 3.3.

Consider a set of successive vertices $S=\left\{v_{1}, v_{2}, \ldots, v_{N}\right\} \subset \mathbb{Z}$ and the corresponding space $L_{2}(S)$. If $b S=\left\{v_{0}, v_{N+1}\right\}$ is the boundary of $S$, then for any $\varphi \in L_{2}(S)$ the function $\mathcal{L}_{\mathbb{Z}} \varphi$ has support on $S \cup b S$ and

$$
\begin{gathered}
\mathcal{L}_{\mathbb{Z}} \varphi\left(v_{0}\right)=-\varphi\left(v_{1}\right), \mathcal{L}_{\mathbb{Z}} \varphi\left(v_{1}\right)=2 \varphi\left(v_{1}\right)-\varphi\left(v_{2}\right), \\
\mathcal{L}_{\mathbb{Z}} \varphi\left(v_{N}\right)=2 \varphi\left(v_{N}\right)-\varphi\left(v_{N-1}\right), \mathcal{L}_{\mathbb{Z}} \varphi\left(v_{N+1}\right)=-\varphi\left(v_{N}\right),
\end{gathered}
$$

and for any other $v_{j}$ with $2 \leq j \leq N-1$,

$$
\mathcal{L}_{\mathbb{Z}} \varphi\left(v_{j}\right)=-\varphi\left(v_{N-1}\right)+2 \varphi\left(v_{j}\right)-\varphi\left(v_{N+1}\right) .
$$

Let $C_{2 N+2}=\Gamma(S)$ be a cycle graph

$$
C_{2 N+2}=\left\{u_{-N-1}, u_{-N}, \ldots, u_{-1}, u_{0}, u_{1}, u_{2}, \ldots, u_{N}, u_{N+1}\right\}
$$

with the following identification:

$$
u_{-N-1}=u_{N+1} .
$$

Thus the total number of vertices in $C_{2 N+2}$ is $2 N+2$. We introduce an embedding of $S \cup b S$ into $C_{2 N+2}$ by the following identification:

$$
v_{0}=u_{0}, v_{1}=u_{1}, \ldots, v_{N}=u_{N}, v_{N+1}=u_{N+1} .
$$

This embedding gives a rise to an embedding of $L_{2}(S)$ into $L_{2}\left(C_{2 N+2}\right)$, namely every $\varphi \in L_{2}(S)$ is identified with a function $F_{\varphi} \in L_{2}\left(C_{2 N+2}\right)$ for which

$$
F_{\varphi}\left(u_{0}\right)=0, F_{f \varphi}\left(u_{1}\right)=\varphi\left(v_{1}\right), \ldots, F_{\varphi}\left(u_{N}\right)=\varphi\left(v_{N}\right), F_{\varphi}\left(u_{N+1}\right)=0,
$$

and also

$$
F_{\varphi}\left(u_{-1}\right)=-\varphi\left(v_{1}\right), \ldots, F_{\varphi}\left(u_{-N}\right)=-\varphi\left(v_{N}\right) .
$$

It is important to note that

$$
\sum_{u \in C_{2 N+2}} F_{\varphi}(u)=0
$$

If $\mathcal{L}_{C}$ is the Laplace operator on the cycle $C_{2 N+2}$, then a direct computation shows that for the vector $F_{\varphi}$ defined above the following is true:

$$
2\|\varphi\|=\left\|F_{\varphi}\right\|, 2\left\|\mathcal{L}_{\mathbb{Z}} \varphi\right\|=\left\|\mathcal{L}_{C} F_{\varphi}\right\|, \varphi \in L_{2}(S), F_{\varphi} \in L_{2}\left(C_{2 N+2}\right) .
$$

The operator $\mathcal{L}_{C}$ in $L_{2}\left(C_{2 N+2}\right)$ has a complete system of orthonormal eigenfunctions

$$
\psi_{n}(k)=\exp 2 \pi i \frac{n}{2 N+2} k, 0 \leq n \leq 2 N+1,1 \leq k \leq 2 N+2,
$$

with eigenvalues

$$
\lambda_{n}=1-\cos \frac{2 \pi n}{2 N+2}, 0 \leq n \leq 2 N+1 .
$$

The definition of the function $F_{\varphi} \in L_{2}\left(C_{2 N+2}\right)$ implies that it is orthogonal to all constants and its Fourier series does not contain a term which corresponds to the index $n=0$. It allows us to obtain the following estimate:

$$
\left\|\mathcal{L}_{C} F_{\varphi}\right\|^{2}=\sum_{n=1}^{2 N+1} \lambda_{n}^{2}\left|\left\langle F_{\varphi}, \psi_{n}\right\rangle\right|^{2} \geq 4 \sin ^{4} \frac{\pi}{2 N+2}\left\|F_{\varphi}\right\|^{2} .
$$


It gives the following estimate for functions $f$ from $L_{2}(S)$ :

$$
\|\varphi\| \leq \frac{1}{2} \sin ^{-2} \frac{\pi}{2 N+2}\left\|\mathcal{L}_{\mathbb{Z}} \varphi\right\| .
$$

Thus we proved the following lemma.

Lemma 5.3. If $S=\left\{v_{1}, v_{2}, \ldots, v_{N}\right\}$ consists of $|S|=N$ successive vertices of a line graph $\mathbb{Z}$, then it is a $\Lambda$-set for

$$
\Lambda=\frac{1}{2} \sin ^{-2} \frac{\pi}{2|S|+2} .
$$

In other words, for any $\varphi \in L_{2}(S)$ the following inequality holds true:

$$
\|\varphi\| \leq \Lambda\left\|\mathcal{L}_{\mathbb{Z}} \varphi\right\|
$$

Note that in the case $|S|=1$ the last lemma gives the inequality

$$
\left\|\delta_{v}\right\| \leq\left\|\mathcal{L}_{\mathbb{Z}} \delta_{v}\right\|, S=\{v\}
$$

but direct calculations in Example 1 give a better value for $\lambda$ :

$$
\left\|\delta_{v}\right\|=\sqrt{\frac{2}{3}}\left\|\mathcal{L}_{\mathbb{Z}} \delta_{v}\right\|, v \in V
$$

A combination of this lemma with Lemma 3.7 and Theorem 3.2 gives Theorem 1.4 from the Introduction.

A similar result holds true for a lattice $\mathbb{Z}^{n}$ of any dimension. Consider for example the case $n=2$. In this situation the Fourier transform $\mathcal{F}$ on the space $L_{2}\left(\mathbb{Z}^{2}\right)$ is the unitary operator $\mathcal{F}$ which is defined by the formula

$$
\mathcal{F}(f)\left(\xi_{1}, \xi_{2}\right)=\sum_{\left(k_{1}, k_{2}\right) \in \mathbb{Z}^{2}} f\left(k_{1}, k_{2}\right) e^{i k_{1} \xi_{1}+i k_{2} \xi_{2}}, f \in L_{2}(\mathbb{Z} \times \mathbb{Z}),
$$

where $\left(\xi_{1}, \xi_{2}\right) \in[-\pi, \pi) \times[-\pi, \pi)$. The operator $\mathcal{F}$ is an isomorphism of the space $L_{2}(G)$ on the space $L_{2}(\mathbb{T} \times \mathbb{T})=L_{2}\left(\mathbb{T} \times \mathbb{T}, d \xi_{1} d \xi_{2} / 4 \pi^{2}\right)$, where $\mathbb{T}$ is the one-dimensional torus. The following formula holds true:

$$
\mathcal{F}(\mathcal{L} f)(\xi)=\left(\sin ^{2} \frac{\xi_{1}}{2}+\sin ^{2} \frac{\xi_{2}}{2}\right) \mathcal{F}(f)(\xi)
$$

We have the following result.

Theorem 5.4. The spectrum of the Laplace operator on the lattice $\mathbb{Z}^{2}$ is the set $[0,2]$. A function $f$ belongs to the space $P W_{\omega}(\mathbb{Z} \times \mathbb{Z}), 0<\omega<2$, if and only if the support of $\mathcal{F} f$ is a subset $\Omega_{\omega}$ of $[-\pi, \pi) \times[-\pi, \pi)$ on which

$$
\sin ^{2} \frac{\xi_{1}}{2}+\sin ^{2} \frac{\xi_{2}}{2} \leq \omega
$$

The same proof as in the case of the one-dimensional lattice gives the following theorem.

Theorem 5.5. If graph $G$ is the 2-dimensional lattice $\mathbb{Z}^{2}$, then for any finite set of vertices $S \subset \mathbb{Z}^{2}$, any $\varphi \in L_{2}(S)$ and any $0<\omega<2$ there exists a function $f_{\varphi} \in P W_{\omega}\left(\mathbb{Z}^{2}\right)$ which coincides with $\varphi$ on $S$. 
Given a set $S=\left\{v_{n, m}\right\}, 1 \leq n \leq N, 1 \leq m \leq M$, we consider the embedding of $S$ into a two-dimensional discrete torus of the size $T=(2 N+2) \times(2 M+2)=\left\{u_{n, m}\right\}$. Every $f \in L_{2}(S)$ is identified with a function $g \in L_{2}(T)$ in the following way:

$$
g\left(u_{n, m}\right)=f\left(v_{n, m}\right), 1 \leq n \leq N, 1 \leq m \leq M,
$$

and

We have

$$
g\left(u_{n, m}\right)=0, N<n \leq N+2, M<m \leq M+2 .
$$

$$
\left\|\mathcal{L}_{G} f\right\|=\left\|\mathcal{L}_{T} g\right\|
$$

where $\mathcal{L}_{T}$ is the combinatorial Laplacian on the discrete torus $T$. Since eigenfunctions of $\mathcal{L}_{T}$ are products of the corresponding functions (5.1) a direct calculation gives the following inequality:

$$
\|\varphi\| \leq \frac{1}{4} \frac{1}{\min \left(\sin \frac{\pi}{2 N+2}, \sin \frac{\pi}{2 M+2}\right)}\left\|\mathcal{L}_{G} \varphi\right\|, \varphi \in L_{2}(S) .
$$

As a consequence we obtain Theorem 1.5 from the Introduction.

We now turn to homogeneous trees. On homogeneous trees there is a well developed harmonic analysis [12, [5]. In particular there is the Helgason-Fourier transform which provides the spectral resolution of the combinatorial Laplacian $\mathcal{L}$. One can use this Helgason-Fourier transform to give a more explicit definition of the Paley-Wiener spaces $P W_{\omega}(G)$.

We briefly introduce the spherical harmonic analysis on homogeneous trees. Let $G$ be a homogeneous tree of order $q+1, q \geq 2$ and $o$ be a root of it. The distance of $v \in V(G)$ from $o$ is denoted by $|v|$. A function in $L_{2}(G)$ is said to be radial if it depends only on $|v|$. The space of all radial functions in $L_{2}(G)$ will be denoted by $L_{2}^{\natural}(G)$, and the space of all radial functions in $P W_{\omega}(G)$ will be denoted by $P W_{\omega}^{\natural}(G)$.

We introduce the notation $\tau=2 \pi / \ln q$ and consider the torus $\mathbb{T}=\mathbb{R} / \tau \mathbb{Z}$ which is identified with $[-\tau / 2, \tau / 2)$. Let $\mu$ denote the Plancherel measure on $\mathbb{T}$, given by the formula

where

$$
d \mu(\xi)=\frac{q \ln q}{4 \pi(q+1)}|\mathbf{c}(\xi)|^{-2} d \xi,
$$

$$
\mathbf{c}(\xi)=\frac{q^{1 / 2}}{q+1} \frac{q^{1 / 2+i \xi}-q^{-1 / 2-i \xi}}{q^{i \xi}-q^{-i \xi}}, \xi \in \mathbb{T} .
$$

The spherical functions $\Phi_{\xi}(v), \xi \in[-\tau / 2, \tau / 2), v \in V(G)$, are the radial eigenfunctions of the Laplace operator $\mathcal{L}$ satisfying $\Phi_{\xi}(v)(o)=1$. The explicit formula for such functions is

$$
\mathbf{c}(\xi) q^{(i \xi-1 / 2)|v|}+\mathbf{c}(\xi) q^{(-i \xi-1 / 2)|v|}, \xi \in[-\tau / 2, \tau / 2), v \in V(G),
$$

and the corresponding eigenvalue is given by the formula

$$
1-\eta(q) \cos (\xi \ln q), \eta(q)=\frac{2 q^{1 / 2}}{q+1} .
$$

The spherical Helgason-Fourier transform is defined by the formula

$$
\mathcal{H} f(\xi)=\sum_{v \in V(G)} f(v) \Phi_{\xi}(v), \xi \in[-\tau / 2, \tau / 2), f \in L_{1}^{\natural}(G) .
$$

The following theorem is known 12 . 
Theorem 5.6. The spherical Helgason-Fourier transform extends to an isometry of $L_{2}^{\natural}(G)$ onto $L_{2}(\mathbb{T}, \mu)$, and the corresponding Plancherel formula holds

$$
\|f\|=\left(\int_{-\tau / 2}^{\tau / 2}|\mathcal{H}(f)(\xi)|^{2} d \mu(\xi)\right)^{1 / 2}, f \in L_{2}^{\natural}(G) .
$$

Using the fact that $\Phi_{z}(v), v \in V(G)$, is an eigenfunction of $\mathcal{L}$ with the eigenvalue (5.3), one can obtain the following formula:

$$
\mathcal{H}(\mathcal{L} f)(\xi)=(1-\eta(q) \cos (\xi \ln q)) \mathcal{H}(f)(\xi) .
$$

The next statement is obvious.

Theorem 5.7. If $G$ is a homogeneous tree of order $q+1$, then a function $f$ belongs to the space $P W_{\omega}^{\natural}(G)$ if and only if the support of $\mathcal{H} f$ is a subset $\Pi_{\omega}$ where

$$
\Pi_{\omega}=\{\xi \in[1-\eta(q), 1+\eta(q)]: 1-\eta(q)<1-\eta(q) \cos (\xi \ln q) \leq \omega\} .
$$

To formulate the next theorem we introduce the restriction operator

$$
\mathcal{R}_{\omega}^{S}: P W_{\omega}^{\natural}(G) \rightarrow L_{2}^{\natural}(S), S \subset V(G),
$$

where

$$
\mathcal{R}_{\omega}^{S}(\varphi)=\left.\varphi\right|_{S}, \varphi \in P W_{\omega}^{\natural}(G) .
$$

Theorem 5.8. If $G$ is a homogeneous tree, then for every $\omega>0$ and every finite set $S \subset V(G)$ the restriction operator $\mathcal{R}_{\omega}^{S}$ is surjective.

Proof. Pick a finite set $S$ and assume that a function $\psi \in L_{2}^{\natural}(S)$ is orthogonal to all restrictions to the set $S$ of all functions from a space $P W_{\omega}^{\natural}(G), 1-\eta(q)<\omega<$ $1+\eta(q)$. It is known [5] that the functions $z \rightarrow \Phi_{z}(v)$ are entire functions for every fixed $v \in V(G)$. Since $\psi$ has support on a finite set $S$ its Helgason-Fourier transform $\mathcal{H}$ according to the formula (5.1) is a finite combination of some functions $\Phi_{\xi}(v), \xi \in[-\tau / 2, \tau / 2), v \in V(G)$, and in particular an analytic function on the real line. It implies that the set of zeros of $\mathcal{H} \psi$ has measure zero. At the same time by the Parseval's relation this function should be orthogonal to any function with support in the set $\Pi_{\omega}$ which is a set of positive measure. This shows that there is no function in $L_{2}^{\natural}(S)$ which is orthogonal to restrictions to $S$ of all functions in $P W_{\omega}^{\natural}(G)$. Because the set $L_{2}^{\natural}(S)$ is finite-dimensional it implies that it is exactly the space of all restrictions of $P W_{\omega}^{\natural}(G)$ to $S$. The theorem is proved.

On homogeneous trees of order $q+1, q \geq 2$, the spectrum of Laplacian $\mathcal{L}$ is separated from zero, and in this case the operator $\mathcal{L}^{s}, s \in \mathbb{R}$, can be used as the operator $\mathcal{B}$ from the corollary with

$$
\left\|\mathcal{L}^{s}\right\|=\omega^{s},\left\|\mathcal{L}^{-s}\right\|=(1-\eta(q))^{-s}, q \geq 2, s \in \mathbb{R} .
$$

The corresponding inequality with $\mathcal{L}^{s}, s \in \mathbb{R}$, in place of $\mathcal{B}$ means that a function $f \in P W_{\omega}(G)$ is uniquely determined by the values of its "derivatives" $\mathcal{L}^{s} f, s \in \mathbb{R}$, on a uniqueness set $U$.

Theorem 5.9. If $G$ is a homogeneous tree, then for any $s \in \mathbb{R}$ there exists a frame $\left\{\Psi_{u}^{(s)}\right\}_{u \in U}$ in the space $P W_{\omega}(G)$ such that the following reconstruction formula holds true for all $f \in P W_{\omega}(G)$ :

$$
f(v)=\sum_{u \in U} \mathcal{L}^{s} f(u) \Psi_{u}^{(s)}(v), v \in V(G) .
$$


In what follows we will obtain explicit constants for the Poincare inequality for some specific finite sets of point on homogeneous trees. What is interesting is that these sets have a large volume and can even be infinite, but corresponding Poincare constants are less than one.

Consider a homogeneous tree of order $q+1$. We will say that the root of this tree belongs to the level zero, the next $q$ vertices belong to the level one, the next $q^{2}$ belong to the level two, and so on. A level of order $m$ will be denoted as $l_{m}$. Direct computations show that the following lemma holds true.

Lemma 5.10. On a homogeneous tree $G$ of order $q$ for any level $S=l_{m}$ of order $m$ the following Poincare inequality holds true:

$$
\|\varphi\| \leq\left(1+\frac{q}{(q+1)^{2}}\right)^{-1 / 2}\|\mathcal{L} \varphi\|, \varphi \in L_{2}\left(l_{m}\right) .
$$

It implies in particular that any finite or infinite set of the form

$$
S=\bigcup_{m=0} l_{3 m}
$$

is a removable set for any space $P W_{\omega}(G)$ with any $\omega<\alpha(q)=\left(1+\frac{q}{(q+1)^{2}}\right)^{1 / 2}>1$.

Moreover, there exists a frame of functions $\left\{\Theta_{u}\right\}_{u \in U}, U=V(G) \backslash S$, in the space $P W_{\omega}(G)$ such that the following reconstruction formula holds true for all $f \in P W_{\omega}(G):$

$$
f(v)=\sum_{u \in U} f(u) \Theta_{u}(v), v \in V(G)
$$

Another way to reconstruct $f \in P W_{\omega}(G)$ is by using Theorem 1.4 with

$$
\Lambda=\left(1+\frac{q}{(q+1)^{2}}\right)^{-1 / 2} .
$$

Note that according to Lemma 3.5 for all functions in $P W_{\omega}(G)$ where $\omega<1$, any set of the form

$$
S=\bigcup_{m=0} l_{2 m}
$$

is a removable set.

\section{Applications to Finite GRAphs}

In this section we consider finite graphs. For a set $S \subset V(G)$ with a non-empty boundary $b S$ consider the set $\mathcal{D}(S)$ of all functions $f$ from $L_{2}(G)$ which vanish on the boundary $b S$. For a subset $S \subset V(G)$ the induced subgraph determined by all edges that have both endpoints in $S$. The first Dirichlet eigenvalue of an induced subgraph on $S$ is defined as follows [4]:

$$
\lambda_{D}(S)=\inf _{f \in \mathcal{D}(S), f \neq 0} \frac{\langle f, \mathcal{L} f\rangle}{\langle f, f\rangle} .
$$

Because $L_{2}(S) \subset \mathcal{D}(S)$ we obtain the inequality

$$
\|\varphi\| \leq \frac{1}{\lambda_{D}(S)}\left\|\mathcal{L}_{G} \varphi\right\|, \varphi \in L_{2}(S),
$$

which is different from (3.7). 
Note that since $L_{2}(S)$ is a subspace of $\mathcal{D}(S)$ the constant in inequality (6.2) is not the best possible. Only when $\bar{S}=S \cup b S$ coincides with the entire set $V(G)$ is this inequality exact.

If $\delta$ is the isoperimetric dimension of the graph $G$, then there exists a constant $C_{\delta}$ which depends only on $\delta$ (see [4]) such that

$$
\lambda_{D}(S)>C_{\delta}\left(\frac{1}{\operatorname{vol} S}\right)^{2 / \delta}, \operatorname{vol} S=\sum_{v \in S} d(v) .
$$

We obtain the following statement in which we use the same notation as above.

Theorem 6.1. If a set $S \subset V(G)$ and an $\omega>0$ satisfy the inequality

$$
\omega<C_{\delta}\left(\frac{1}{\operatorname{vol} S}\right)^{2 / \delta}
$$

then the set $S$ is removable for the space $P W_{\omega}(G)$.

The following statement gives a certain connection between the distribution of eigenvalues and the existence of specific sets of vertices.

Theorem 6.2. If a finite graph $G$ has $N=|V(G)|$ vertices and a set $S \subset V(G)$ is a set of type $\Lambda$, then there are at most $|U|$ eigenvalues (with multiplicities) of $\mathcal{L}$ on the interval $[0,1 / \Lambda)$ where $U=V \backslash S$ and there are at least $N-|U|$ eigenvalues which belong to the interval $\left[1 / \Lambda, \lambda_{\max }\right]$.

Proof. If $S$ is a set of type $\Lambda$, then $U=V \backslash S$ is a uniqueness set for any space $P W_{\omega}(G)$ with $\omega<1 / \Lambda$. It means that $|U|$, which is the dimension of the space $L_{2}(U)$, cannot be less than the number of eigenvalues (with multiplicities) of $\mathcal{L}$ on the interval $[0,1 / \Lambda)$.

Theorem 6.2 implies Corollaries 1.1 and 1.2 from the Introduction.

\section{ACKNOWLEDGEMENT}

The author would like to thank Dr. Meyer Pesenson for encouragement and many useful conversations.

\section{REFERENCES}

[1] A. Beurling, Local harmonic analysis with some applications to differential operators, Some Recent Advances in the Basic Sciences, vol. 1, Belfer Grad. School Sci. Annu. Sci. Conf. Proc., A. Gelbart, ed., 1963-1964, 109-125. MR0427956 (55:986)

[2] A. Beurling and P. Malliavin, On the closure of characters and the zeros of entire functions, Acta Math., 118 (1967), 79-95. MR0209758 (35:654)

[3] M. Birman and M. Solomyak, Spectral theory of selfadjoint operators in Hilbert space, D. Reidel Publishing Co., Dordrecht, 1987. MR1192782 (93g:47001)

[4] F. R. K. Chung, Spectral Graph Theory, CBMS 92, AMS, 1994.

[5] M. Cowling, S. Meda, A. Setti, An overview of harmonic analysis on the group of isometries of a homogeneous tree, Exposition. Math., 16 (1998), 385-423. MR.1656839 (2000i:43005)

[6] R. Duffin, A. Schaeffer, A class of nonharmonic Fourier series, Trans. AMS, 72 (1952), 341-366. MR0047179(13:839a)

[7] M. Ebata, M. Eguchi, S. Koizumi, K. Kumahara, On sampling formulas on symmetric spaces, J. Fourier Anal. Appl., 12 (2006), no. 1, 1-15. MR2215673

[8] M. Ebata, M. Eguchi, S. Koizumi, K. Kumahara, Analogues of sampling theorems for some homogeneous spaces, Hiroshima Math. J., 36 (2006), no. 1, 125-140. MR2213647 (2007h:43004) 
[9] K. Fan, O. Taussky, J. Todd, Discrete analogs of inequaliyies of Wirtinger, Monatsh. fur Mathematik, 59 (1955), 73-90. MR0070676 (17:19b)

[10] H. Feichtinger and I. Pesenson, Iterative recovery of band limited functions on manifolds, in Wavelets, Frames and Operator Theory, Contemp. Math., 345, AMS, (2004) 137-153. MR2066825 (2006d:43008)

[11] H. Feichtinger and I. Pesenson, A reconstruction method for band-limited signals on the hyperbolic plane, Sampl. Theory Signal Image Process., 4 (2005), no. 2, 107-119. MR2138377 (2006k:94016)

[12] A. Figa-Talamanca, C. Nebbia, Harmonic Analysis and Representation Theory for Groups Acting on Homogeneous Trees, London Math. Soc. Lecture Notes Series, 162, Cambridge Univ. Press, Cambridge, 1991. MR.1152801 (93f:22004)

[13] M.W. Frazier, R. Torres, The sampling theorem, $\varphi$-transform, and Shannon wavelets for $\mathbb{R}, \mathbb{Z}, \mathbb{T}$, and $\mathbb{Z}_{N}$. Wavelets: Mathematics and Applications, 221-246, Stud. Adv. Math., CRC, Boca Raton, FL, 1994. MR.1247518 (94k:42049)

[14] H. Führ, Abstract Harmonic Analysis of Continuous Wavelet Transforms, Lecture Notes in Mathematics, 1863, Springer, 2005. MR.2130226 (2006m:43003)

[15] H. Führ and K. Gröchenig, Sampling theorems on locally compact groups from oscillation estimates, Math. Z., 255 (2007), no. 1, 177-194. MR2262727

[16] K. Gröchenig, A Discrete Theory of Irregular Sampling, Linear Algebra and its Applications, 193 (1993), 129-150. MR 1240276 (94m:94005)

[17] G. H. Hardy, J. E. Littlewood, G. Polya, Inequalities, Cambridge, England, 1952. MR0046395 $(13: 727 \mathrm{e})$

[18] G. T. Herman, A. Kuba, Discrete Tomography, Foundations, Algorithms, and Applications, Birkhauser, 1999. MR1722457 (2000h:92015)

[19] H. Landau, Necessary density conditions for sampling and interpolation of certain entire functions, Acta. Math., 117 (1967), 37-52. MR0222554 (36:5604)

[20] Y. Lyubarskii, K. Seip, Weighted Paley-Wiener spaces, J. Amer. Math. Soc., 15 (2002), no. 4, 979-1006. MR1915824 (2003m:46039)

[21] A. Magyar, E. M. Stein, S. Wainger, Discrete analogues in harmonic analysis: spherical averages, Ann. of Math. (2), 155 (2002), no. 1, 189-208. MR1888798(2003f:42028)

[22] B. Mohar, The Laplace spectrum of graphs, "Graph Theory, Combinatorics, and Applications", Vol. 2, Wiley, 1991, 871-898. MR1170831 (93b:05116)

[23] J. Ortega-Cerda, K. Seip, Fourier frames, Annals of Math., 155 (2002), 789-806. MR1923965 (2003k:42055)

[24] R.E.A.C. Paley and N. Wiener, Fourier Transforms in the Complex Domain, Coll. Publ., 19, Providence: Amer. Math. Soc. (1934). MR1451142 (98a:01023)

[25] I. Pesenson, Sampling of Paley-Wiener functions on stratified groups, J. of Fourier Analysis and Applications, 4 (1998), 269-280. MR1650917 (99j:41024)

[26] I. Pesenson, Reconstruction of band-limited functions in $L_{2}\left(R^{d}\right)$, Proceed. of AMS, 127 (12), (1999), 3593-3600. MR1610773 (2000f:42002)

[27] I. Pesenson, A sampling theorem on homogeneous manifolds, Trans. of AMS, 352 (9), (2000), 4257-4270. MR 1707201 (2000m:41012)

[28] I. Pesenson, Poincaré-type inequalities and reconstruction of Paley-Wiener functions on manifolds, J. of Geometric Analysis 4 (1), (2004), 101-121. MR2030577 (2004h:42030)

[29] I. Pesenson, Deconvolution of band limited functions on symmetric spaces, Houston J. of Math., 32, (2006), no. 1, 183-204. MR2202361 (2007c:43010)

[30] I. Pesenson, Band limited functions on quantum graphs, Proc. Am. Math. Soc. 133, (2005), no. 12, 3647-3655. MR2163603 (2006k:94051)

[31] I. Pesenson, Frames for spaces of Paley-Wiener functions on Riemannian manifolds, in Integral Geometry and Tomography, Contemp. Math., 405, AMS (2006), 137-153. MR2239176 (2007k:43016)

[32] I. Pesenson, Analysis of band-limited functions on quantum graphs, Appl. Comput. Harmon. Anal., 21 (2006), no. 2, 230-244. MR.2259780

[33] M. Plancherel, G. Polya, Fonctions entieres et integrales de Fourier multiples, Comment. Math. Helv., 9 (1937), 224-248.

[34] M. Plancherel, G. Polya, Fonctions entieres et integrales de Fourier multiples, Comment. Math. Helv., 10 (1938), 110-163. MR.1509570 
[35] D. E. Rutherford, Some continuant determinants arising in physics and chemistry, I, Proc. Royal Soc. Edinburg, 62A (1947), 229-236; II, 63A (1952), 232-241. MR0020070 (8:499b)

[36] S. Smale, D. X. Zhou, Shannon sampling. II. Connections to learning theory, Appl. Comput. Harmon. Anal., 19 (2005), no. 3, 285-302. MR2186447 (2006i:94024)

[37] S. Smale, D. X. Zhou, Shannon sampling and function reconstruction from point values, Bull. Amer. Math. Soc. (N.S.), 41 (2004), no. 3, 279-305. MR.2058288(2005b:94022)

[38] H. Triebel, Theory of function spaces II, Monographs in Mathematics, 84. Birkhäuser Verlag, Basel, 1992. MR.1163193 (93f:46029)

[39] A.I. Zayed, Advances in Shannon's Sampling Theory, CRC Press, Boca Raton, 1993. MR.1270907 (95f:94008)

Department of Mathematics, Temple University, Philadelphia, Pennsylvania 19122

E-mail address: pesenson@temple.edu 